\title{
Aloeresin I, an Anti-Inflammatory 5-Methylchromone from Cape Aloe
}

G. Speranza ${ }^{1}$, C. F. Morelli', A. Tubaro², G. Altinier², L. Durì', P. Manitto ${ }^{1}$

\begin{abstract}
A new diglucoside having a 5-methylchromone moiety was isolated from a commercial sample of Cape aloe, the dried exudate from Aloe ferox Miller, and named aloeresin I. Its structure was established as $\mathbf{1}$ on the basis of spectral and chemical evidence. Aloeresin I (1) $\left(1 \mu \mathrm{mol} / \mathrm{cm}^{2}\right)$ reduces in vivo the oedematous response (39\%) induced by Croton oil in the mouse ear with the same potency as aloesin, one of the most abundant Cape aloe constituents, and to a higher extent than aloeresin $\mathrm{H}$ (2). Indomethacin $\left(0.3 \mu \mathrm{mol} / \mathrm{cm}^{2}\right)$, the reference anti-inflammatory compound, provokes $61 \%$ oedema inhibition.
\end{abstract}

5-Methylchromones are common metabolites of Aloe spp. [1]. Typically, they bear a glucosyl residue at the 8-position which, in turn, is frequently acylated with cinnamoyl residues [1]. Some of them exhibit significant anti-inflammatory [2], [3] and antioxidant [3], [4] activities, thus stimulating the search for new biologically active chromones from natural sources [5]. Continuing our studies on Cape aloe, the dried latex from the leaves of Aloe ferox Mill. [6], we describe here the isolation, structural elucidation and evaluation of the anti-inflammatory activity of a new diglucoside having a 5-methylchromone moiety, named aloeresin I (1).

Aloeresin I (1) was isolated from a commercial sample of Cape aloe in ca. $0.15 \%$ yield. Its mono- and bidimensional ${ }^{1} \mathrm{H}$ - and $13 \mathrm{C}-\mathrm{NMR}$ spectra (Table $\mathbf{1}$ ) were strongly reminiscent of those of aloeresin $\mathrm{H}$ (2) recently isolated from Cape aloe [5]. Additional signals, assignable to an $(E)$-p-coumaroyl group, were also present. That the $p$ coumaroyl group was involved in an ester linkage at the $0-2$ position of the $\mathrm{C}-19$ glucose could be inferred from the marked "acylation effect" observed on the chemical shifts of $\mathrm{H}-2$ ", $\mathrm{C}-1$ ", C-2", and C-3" [7] with respect of those of aloeresin $\mathrm{H}$ (2) [5] (Table 1). The Dconfiguration of the glucose units in $\mathbf{1}$ was deduced from the basecatalysed hydrolysis to give aloeresin $\mathrm{H}(\mathbf{2})$ which, in turn, was chemically related to aloesin [5] (conformational studies of aloesin derivatives based on NOE experiments and CD spectra indicated the $\mathrm{D}$-configuration of glucose in aloesin [8]).

Affiliation: ${ }^{1}$ Dipartimento di Chimica Organica e Industriale, Università degli Studi di Milano, Italy $\cdot{ }^{2}$ Dipartimento di Economia e Merceologia, Università degli Studi di Trieste, Trieste, Italy

Correspondence: Giovanna Speranza · Dipartimento di Chimica Organica e Industriale · Università degli Studi di Milano • via Venezian 21 • 20133 Milano . Italy · Phone: +39-2-5031-4097 - Fax: +39-2-5031-4072 - E-mail: giovanna. speranza@unimi.it

Received: April 6, $2004 \cdot$ Accepted: June 27, 2004

Bibliography: Planta Med 2005; 71: 79-81 • (c) Georg Thieme Verlag KG Stuttgart · New York · DOI 10.1055/s-2005-837756 · ISSN 0032-0943 


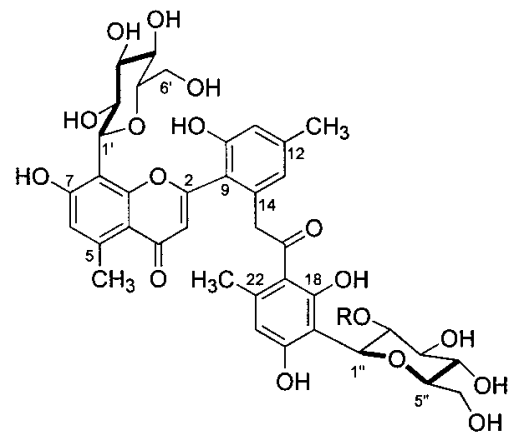<smiles>[R]C(=O)/C=C/c1ccc(O)cc1</smiles>

The topical anti-inflammatory activity of aloeresin I (1) was evaluated using the Croton oil ear test in mice [9] in comparison with that of aloeresin H (2) and that of aloesin [10]. The three chromones were found to possess significant anti-inflammatory activities (Table 2), although lower than that of the NSA reference drug, indomethacin. Aloesin and aloeresin I (1) showed similar anti-inflammatory activity, while aloeresin $\mathrm{H}(\mathbf{2})$, devoid of the p-coumaroyl group, exhibited a slightly inferior activity. Concerning aloesin, it can be noticed that its in vivo anti-inflammatory activity had not been reported till now, although this chromone represents one of the most abundant constituents of Cape aloe (ca. $20 \%$ of the drug) [11]. The mechanism of anti-inflammatory action of aloesin remains to be defined since previous in vitro studies [3] did not give significant and clear information: the reported radical scavenging activity [3] seems to be too moderate to completely justify the in vivo observed anti-inflammatory properties.

\section{Materials and Methods}

1H- and 13C-NMR spectra: Bruker Avance 400 spectrometer; HR ESI MS: Bruker Daltonics FT-ICR APEX-II instrument. Aloesin was isolated from Cape aloe as described in [7].

The commercial Cape aloe used in this investigation was purchased from D. Ulrich Spa (Nichelino, Italy). It was produced in the Port Elisabeth region (Cape Town, South Africa). A voucher specimen (NP-CA 036) is kept at the Dipartimento di Chimica Organica e Industriale, Università di Milano.

Powdered Cape aloe (350 g) was extracted with EtOAc- $\mathrm{CHCl}_{3}-$ $\mathrm{MeOH}$ as previously described [5]. The extract (40 g) was fractionated by flash chromatography (silica gel 230-400 mesh, $1 \mathrm{~kg}$, $8 \times 40 \mathrm{~cm}$ column, EtOAc-MeOH, $1: 1,750 \mathrm{~mL}$ fractions). Fractions 3-6 were combined and concentrated under vacuum. The residue (ca. $15 \mathrm{~g}$ ) was further fractionated by flash chromatography (silica gel, $750 \mathrm{~g}, 8 \times 30 \mathrm{~cm}$ column, $\mathrm{CHCl}_{3}-\mathrm{MeOH}$ gradient from $4: 1$ to $1: 4,200 \mathrm{~mL}$ fractions). Fractions $8-12(2.2 \mathrm{~g})$ were flash chromatographed (silica gel, $300 \mathrm{~g}, 5 \times 30 \mathrm{~cm}$ column, $25 \mathrm{~mL}$ fractions) eluting with EtOAc-EtOH- $\mathrm{H}_{2} \mathrm{O}, 100: 20: 13$, to give aloere$\sin \mathrm{I}(\mathbf{1})$ (fractions $11-23,800 \mathrm{mg}$ ) showing ca. $90 \%$ purity by TLC (silica gel 60 F254 aluminium sheet, Merck, EtOAc-EtOH- $\mathrm{H}_{2} \mathrm{O}$, $100: 20: 13$, Rf: 0.48). Final purification was achieved on a Sephadex LH-20 column $(4 \times 30 \mathrm{~cm})$ using $\mathrm{MeOH}-\mathrm{H}_{2} \mathrm{O}, 1: 1$ as eluent $(1$
Table 1 NMR data of aloeresin I (1) in DMSO- $d_{6}$ at $400 \mathrm{MHz}\left({ }^{1} \mathrm{H}\right)$ and $100 \mathrm{MHz}\left({ }^{13} \mathrm{C}\right)^{\mathrm{a}, \mathrm{b}}$

\begin{tabular}{|c|c|c|c|}
\hline Position & $\delta_{H}(\mathrm{~J}, \mathrm{~Hz})$ & $\delta_{C}$ & $H M B C(H \rightarrow C)$ \\
\hline 2 & & 159.8 & \\
\hline 3 & $6.02 \mathrm{~s}$ & $114.1^{c}$ & $C-2, C-4 a$ \\
\hline 4 & & 178.2 & \\
\hline $4 a$ & & 114.9 & \\
\hline 5 & & $139.9^{d}$ & \\
\hline Me-5 & $2.69 \mathrm{~s}$ & 21.8 & $C-4 a, C-5, C-6$ \\
\hline 6 & $6.67 \mathrm{~s}$ & 116.4 & C-4a, Me-5, C-8 \\
\hline 7 & & 159.4 & \\
\hline 8 & & 110.5 & \\
\hline $8 a$ & & $155.1^{\mathrm{e}}$ & \\
\hline 9 & & 118.4 & \\
\hline 10 & & $155.9^{e}$ & \\
\hline 11 & $6.69 \mathrm{~s}$ & 114.8 & C-9, Me-12, C-13 \\
\hline 12 & & $140.0^{\mathrm{d}}$ & \\
\hline Me-12 & $2.24 \mathrm{~s}$ & 20.6 & $\mathrm{C}-11, \mathrm{C}-12, \mathrm{C}-13$ \\
\hline 13 & $6.52 \mathrm{~s}$ & 121.9 & \\
\hline 14 & & 134.8 & \\
\hline 15 & $\begin{array}{l}4.08,4.13, A B \text { system } \\
(16.8)\end{array}$ & 48.3 & \\
\hline 16 & & 202.4 & \\
\hline 17 & & 120.3 & \\
\hline 18 & & $156.8^{e}$ & \\
\hline 19 & & 107.6 & \\
\hline 20 & & $157.9^{e}$ & \\
\hline 21 & $6.07 \mathrm{~s}$ & 109.3 & $\mathrm{C}-17, \mathrm{C}-19, \mathrm{Me}-22$ \\
\hline 22 & & 136.9 & \\
\hline Me-22 & $1.83 \mathrm{br} \mathrm{s}$ & 19.3 & \\
\hline $1^{\prime}$ & $4.71 \mathrm{~d}(9.8)$ & 73.7 & $C-8, C-2^{\prime}, C-3^{\prime}$ \\
\hline $2^{\prime}$ & $3.84 \mathrm{dd}(9.8)$ & 71.0 & \\
\hline $3^{\prime}$ & $3.14-3.21 \mathrm{~m}$ & 78.3 & \\
\hline $4^{\prime}$ & $3.42-3.50 \mathrm{~m}$ & $69.9^{f}$ & \\
\hline $5^{\prime}$ & $3.35-3.40 \mathrm{~m}$ & 81.2 & \\
\hline $6^{\prime}$ & $\begin{array}{l}3.61-3.68 \mathrm{~m} ; 3.73 \mathrm{dd} \\
(11.8,2.0)\end{array}$ & $60.1^{9}$ & \\
\hline $1^{\prime \prime}$ & $4.95 \mathrm{~d}(10.0)$ & 72.5 & $C-19, C-2^{\prime \prime}, C-3^{\prime \prime}$ \\
\hline $2^{\prime \prime}$ & $5.17 \mathrm{dd}(10.0)$ & 72.4 & \\
\hline $3^{\prime \prime}$ & $3.55 \mathrm{dd}(10.0)$ & 75.4 & \\
\hline $4^{\prime \prime}$ & $3.42-3.50 \mathrm{~m}$ & $69.6^{f}$ & \\
\hline $5^{\prime \prime}$ & $3.14-3.21 \mathrm{~m}$ & 80.8 & \\
\hline $6^{\prime \prime}$ & $\begin{array}{l}3.61-3.68 \mathrm{~m} \\
3.42-3.50 \mathrm{~m}\end{array}$ & $60.9^{9}$ & \\
\hline $1^{\prime \prime \prime}$ & & 164.8 & \\
\hline $2^{\prime \prime \prime}$ & $6.16 \mathrm{~d}(15.9)$ & $114.3^{c}$ & $C-1^{\prime \prime \prime}, C-4^{\prime \prime \prime}$ \\
\hline $3^{\prime \prime \prime}$ & $7.40 \mathrm{~d}(15.9)$ & 143.6 & $C-1^{\prime \prime \prime}, C-2^{\prime \prime \prime}, C-5^{\prime \prime \prime}$ \\
\hline $4^{\prime \prime \prime}$ & & 125.0 & \\
\hline $5^{\prime \prime \prime}, 9^{\prime \prime \prime}$ & $7.46 \mathrm{~d}(8.6)$ & 129.5 & $C-3^{\prime \prime \prime}, C-7^{\prime \prime \prime}, C-5^{\prime \prime \prime \prime}$ \\
\hline $6^{\prime \prime \prime}, 8^{\prime \prime \prime}$ & $6.78 \mathrm{~d}(8.6)$ & 115.5 & C-4"', C-7'"' \\
\hline $7^{\prime \prime \prime}$ & & 159.6 & \\
\hline
\end{tabular}

$\delta$ in ppm vs. solvent signal as internal reference (DMSO- $\left.d_{6}: \delta_{\mathrm{H}}=2.50, \delta_{\mathrm{C}}=39.50\right)$; spectra recorded at $50^{\circ} \mathrm{C}$

$b$ Signals of glucose hydroxy groups were observed in the range $\delta=3.0-5.0$ and broad signals of phenolic groups were at $\delta=9.93,9.75,9.40,9.32$.

c. $g$ Signals with the same superscript are interchangeable.

drop/3 s, $10 \mathrm{~mL}$ fractions). Aloeresin I (1) (fractions 35-54, 550 $\mathrm{mg}$ ) was obtained as an amorphous powder, pure by TLC and analytical HPLC (Merck LiChrospher 100 RP-18 column, 
Table 2 Anti-inflammatory activity of aloeresin I (1), aloeresin H (2) and aloesin

\begin{tabular}{|lllll|}
\hline Substance & $\begin{array}{l}\text { Dose } \\
\left(\mu \mathrm{mol} / \mathrm{cm}^{2}\right)\end{array}$ & $\boldsymbol{N}^{\circ}$ an. & $\begin{array}{l}\text { Oedema }(\mathbf{m g}) \\
\text { Mean } \pm \text { S.E. }\end{array}$ & $\begin{array}{l}\text { Oedema } \\
\text { inhibition (\%) }\end{array}$ \\
\hline Controls & - & 10 & $6.9 \pm 0.3$ & - \\
\hline Aloeresin I (1) & 0.3 & 10 & $5.2 \pm 0.3^{*}$ & 25 \\
\hline Aloeresin H (2) & 1.0 & 10 & $4.2 \pm 0.3^{*}$ & 39 \\
\hline & 0.1 & 10 & $6.4 \pm 0.3$ & 7 \\
\hline Aloesin & 0.3 & 10 & $5.6 \pm 0.4^{*}$ & 19 \\
\hline & 1.0 & 10 & $4.8 \pm 0.3^{*}$ & 30 \\
\hline Indomethacin & 0.1 & 10 & $6.4 \pm 0.3^{*}$ & 7 \\
\hline & 0.3 & 10 & $5.3 \pm 0.3$ & 23 \\
\hline
\end{tabular}

${ }^{*} \mathrm{p}<0.05$ at the Student's $t$-test.

$125 \times 4 \mathrm{~mm}, 5 \mu \mathrm{m}$; $\mathrm{MeOH}-\mathrm{H}_{2} \mathrm{O}$ linear gradient from 30 to $90 \%$ $\mathrm{MeOH}$ in $30 \mathrm{~min}, 1 \mathrm{~mL} / \mathrm{min}$; detector, $\lambda=225 \mathrm{~nm}$; Rt: $16.4 \mathrm{~min})$; m.p. (uncorrected) $227-229^{\circ} \mathrm{C}$ (dec); $[\alpha]_{D}^{20}:-91.7^{\circ}$ (c 0.5, MeOH); $\mathrm{UV}(\mathrm{MeOH}): \lambda \max (\log \varepsilon)=212$ (4.72), 226 (4.66), 254 (4.38) 302 (4.57) nm; IR (KBr): vmax = 3392, 2923, 1699, 1648, 1603, 1454, $1380 \mathrm{~cm}^{-1}$; ${ }^{1} \mathrm{H}$ - and ${ }^{13} \mathrm{C}-\mathrm{NMR}$ data, see Table 1 ; HR-ESI-MS: $\mathrm{m} / \mathrm{z}=$ 917.2866280 [M + H] $]^{+}$; calcd. for $\mathrm{C}_{47} \mathrm{H}_{49} \mathrm{O}_{19}$ : 917.2866558.

The topical anti-inflammatory activity was evaluated as inhibition of the Croton oil-induced ear oedema in mice [9]. Male CD1 mice (28-32 g; Harlan-Italy, Udine, Italy) were kept for one week, before the experiment, at constant conditions of temperature $\left(21 \pm 1^{\circ} \mathrm{C}\right)$ and humidity $(60-70 \%)$, with a fixed artificial light cycle (7.00-19.00 h). Inflammation was induced on the inner surface of the right ear (surface: about $1 \mathrm{~cm}^{2}$ ) of mice anaesthetised with ketamine hydrochloride $(145 \mathrm{mg} / \mathrm{kg}$, intraperitoneally; Virbac S.r. 1., Milan, Italy) by application of $80 \mu \mathrm{g}$ of Croton oil (Sigma, St. Louis, Missouri, USA), suspended in $42 \%$ aqueous ethanol $(\mathrm{v} / \mathrm{v})$ together with the test substances; control animals received only the irritant suspension. Aloeresin I (1) was tested at 0.3 and $1.0 \mu \mathrm{mol} / \mathrm{cm}^{2}$, while aloeresin $\mathrm{H} \mathrm{(2)}$ and aloesin were tested at $0.1-0.3-1.0 \mu \mathrm{mol} / \mathrm{cm}^{2}$. As a reference, the non-steroidal anti-inflammatory drug (NSA) indomethacin $\left(0.3 \mu \mathrm{mol} / \mathrm{cm}^{2}\right)$ (Sigma, St. Louis, Missouri, USA) was used. At the maximum of the oedematous response, six hours later, mice were sacrificed and a plug ( $6 \mathrm{~mm} \emptyset$ ) was excised from both the treated (right) and the untreated (left) ears. Oedema was measured as the weight difference between the two plugs. The anti-inflammatory activity was expressed as percent reduction of the oedematous response in treated mice compared to the control mice. All animal experiments complied with the Italian D.L. n. 116 of 27 January 1992 and associated guidelines in the European Communities Council Directive of 24 November 1986 (86/609 ECC).

Pharmacological data were analysed by Student's $t$-test, accepting as significant a probability level lower than 0.05 .

\section{References}

${ }^{1}$ Dagne E, Bisrat D, Viljoen A, Van Wyk B-E. Chemistry of Aloe species. Curr Org Chem 2000; 4: 1055-78

${ }^{2}$ Hutter JA, Salman M, Stavinoha WB, Satsangi N, Williams RF, Streeper RT, Weintraub ST. Anti-inflammatory C-glucosyl chromone from Aloe barbadensis. J Nat Prod 1996; 59: 541 -3

${ }^{3}$ Yagi A, Kabash A, Okamura N, Haraguchi H, Moustafa SM, Khalifa TI. Antioxidant, free radical scavenging and anti-inflammatory effects of aloesin derivatives in Aloe vera. Planta Med 2002; 68: 957-60

${ }^{4}$ Lee KY, Weintraub ST, Yu BP. Isolation and identification of a phenolic antioxidant from Aloe barbadensis. Free Rad Biol \& Med 2000; 28: $261-5$

${ }^{5}$ Manitto P, Speranza G, De Tommasi N, Ortoleva E, Morelli CF. Aloere$\sin \mathrm{H}$, a new polyketide constituent of Cape aloe. Tetrahedron 2003; 59: $401-8$

${ }^{6}$ Tyler VE, Brady LR, Robbers J. Pharmacognosy. Philadelphia: Lee and Fabiger, 1988: pp 62-3

${ }^{7}$ Speranza G, Gramatica P, Dadà G, Manitto P. Aloeresin C, a bitter C,Odiglucoside from Cape aloe. Phytochemistry 1985; 24: 1571 -3

${ }^{8}$ Manitto P, Monti D, Speranza G. Conformational studies of natural products. III. Conformation of natural 8-C-glucosyl-7-hydroxy-5-methylchromones and their derivatives. Gazz Chim Ital 1990; 120: 641 -6

${ }^{9}$ Tubaro A, Dri P, Delbello G, Zilli C, Della Loggia R. The Croton oil ear test revisited. Agents Actions 1985; 19: $347-9$

${ }^{10}$ Haynes LJ, Holdsworth DK, Russell R. C-Glycosyl compounds. Part VI. Aloesin, a C-glucosylchromone from Aloe sp. J Chem Soc (C), 1970: 2581-6

${ }^{11}$ Speranza G, Corti S, Manitto P. Isolation and chemical characterization of a new constituent of Cape aloe having the 1,1-diphenylethane skeleton. J Agric Food Chem 1994; 42: 2002-6

\section{Acknowledgements}

We are grateful to MIUR (Italy) for financial support. 